\title{
Orientation-dependent energy-storage performance and electrocaloric effect in PLZST antiferroelectric thick films
}

Ye Zhao ${ }^{\mathrm{a}, \mathrm{b}}$, Hongcheng $\mathrm{Gao}^{\mathrm{a}}$, Xihong $\mathrm{Hao}^{\mathrm{a}, *}$, Qi Zhang ${ }^{\mathrm{c}, \mathrm{d}}$

${ }^{\text {a }}$ School of Materials and Metallurgy, Inner Mongolia University of Science and Technology, Baotou 014010, China

${ }^{\mathrm{b}}$ Electronic Materials Research Laboratory, Key Laboratory of the Ministry of Education and International Center for Dielectric Research, Xi'an Jiaotong University, Xi'an 710049, China

${ }^{\mathrm{c}}$ State Key Laboratory of Advanced Technology for Materials Synthesis and Processing, Wuhan University of Technology, Wuhan 430070, Hubei, China

d Department of Manufacturing and Materials, Cranfield University, Cranfield, Bedfordshire, MK43 OAL, UK

\section{A b s t r a c t}

The enhancement of the energy-storage performance and electrocaloric effect (ECE) was achieved via orientation control. The 1.5-mm-(Pb0.97La0.02)(Zr0.73Sn0.22Ti0.05)O3 (PLZST) antiferroelectric (AFE) thick films with (111), (110), and (100) crystallographic orientations were successfully prepared via a sol-gel method. It was found that both the enhanced energy-storage density of $13.5 \mathrm{~J} / \mathrm{cm}^{3}$ at $900 \mathrm{kV} / \mathrm{cm}$ and the corresponding temperature reduction of DT $=28.1{ }^{\circ} \mathrm{C}$ at room temperature were obtained in the PLZST thick film with (111) orientation due to a high polarization. Moreover, these films with different orientations display a small leakage current density at the room temperature. These results suggest that both energy-storage properties and cooling performance in AFEs could be optimized by the proper orientation control.

\section{Introduction}

In order to reduce the dependency on fossil based energy reserves, the successful production, distribution, and storage of electricity will form a cornerstone for the development and growth of society and technology in the coming century. The generation of electricity from renewable and non-conventional resources is being investigated and is attracting much attention. High energy-storage density dielectric capacitors as a means for storage of electric energy form an indispensible part of all modern electronic and electrical devices [1-4].

In generasmall remnant polarizationl, the recoverable energy-storage density $\mathrm{W}$ and the energy-storage efficiency h are two crucial parameters, which can be calculated according to the equations as below [5]: 


$$
\begin{aligned}
& W=\int_{P_{r}}^{P_{\max }} E d P, \\
& W_{\text {loss }}=\int E d P, \\
& \eta=\frac{W}{W+W_{\text {loss }}},
\end{aligned}
$$

where $\mathrm{E}$ is the applied electric field, $\mathrm{P}$ is the polarization, $\mathrm{P}_{\mathrm{r}}$ is remanent polarization, $\mathrm{Pmax}$ is the maximum polarization, and Wloss is the energy loss density. Obviously, the high energy-storage density depends on smaller $\mathrm{P}_{\mathrm{r}}$, larger Pmax and good electric field endurance. Antiferroelectrics (AFEs) play a key role in the dielectric energy-storage capacitors due to the high maximum polarization and large electric field induced-strain. In recent years, $\mathrm{PbZrO}$-strain3-based $\mathrm{AFE}$ materials, such as $\mathrm{Pb}_{0.97} \mathrm{La}_{0.02}\left(\mathrm{Zr}_{0.97} \mathrm{Ti}_{0.03}\right) \mathrm{O}_{3}$, $\left(\mathrm{Pb}_{0.97} \mathrm{La}_{0.02}\right)\left(\mathrm{Zr}_{0.95}\right.$. $\left.{ }_{x} \mathrm{Sn}_{\mathrm{x}} \mathrm{Ti}_{0.05}\right) \mathrm{O}_{3}, \mathrm{~Pb}_{0.8} \mathrm{Ba}_{0.2} \mathrm{ZrO}_{3}, \mathrm{Sr}$-doped $\mathrm{PbZrO}_{3}$, Eu-doped $\mathrm{PbZrO}$, and $\mathrm{Nb}$-doped $\mathrm{PbZr} 0.4 \mathrm{Ti} 0.603$, were widely investigated in the high energy-storage performancewidely investigated in the high energy-storage density capacitors storage performance [6-10].

Like dielectric capacitors, a highly efficient and environmentally friendly solid-state cooling technology also has attracted much attention for the micro-electromechanical systems [11,12]. It offers an alternative to the traditional vapor-compression cooling method accompanied hazardous gases, which is widely used in household and industrial applications. There are several technologies being developed by using of various solid-state cooling technology, such as electrocaloric, magnetocaloric and mechano-caloric effects. Electrocaloric effect (ECE) refers to a change in temperature under adiabatic application of external electric fields [13-15]. Alternatively, it can be described as an isothermal change in entropy. To achieve a large electrocaloric effect, a phase transition is desirable, where the entropy change (DS) is maximized. For example, the giant ECE observed in the $0.9 \mathrm{PbMg}_{1 / 3} \mathrm{Nb}_{2 / 3} \mathrm{O}_{3}{ }^{-} 0.1 \mathrm{PbTiO}_{3}$ film, $\mathrm{PbZr}_{0.95} \mathrm{Ti}_{0.05} \mathrm{O}_{3}$ film, and $\mathrm{Pb}$ 
$\left(\mathrm{Zr}_{0.455} \mathrm{Sn}_{0.455} \mathrm{Ti}_{0.09}\right) \mathrm{O}_{3}$ bulk ceramic are associated with ferroelec-tric (FE)-paraelectric (PE), AFE-PE, and AFE-FE phase transitions, respectively [16-18]. Compared with FE (or AFE)-PE phase transition, the FEAFE phase transition could be easily realized in a wide temperature range near $25{ }^{\circ} \mathrm{C}$. Moreover, a considerable pyroelectric effect is also formed during FE-AFE transition, which is even larger than that caused by FE (or AFE)-PE phase transition. Therefore, good ECE should also be obtained during FE-AFE phase transition. Quite recently, a giant ECE $\left(\mathrm{DT}=53.8{ }^{\circ} \mathrm{C}\right.$ and $\mathrm{DS}=63.9 \mathrm{~J} \mathrm{~K}^{-1} \mathrm{~kg}^{-1}$ ) with an electrocaloric coefficient of $0.060 \mathrm{~K} \mathrm{~cm} / \mathrm{kV}$ was also achieved at $5{ }^{\circ} \mathrm{C}$ in our work on $2-\mu \mathrm{m}-$ $\mathrm{Pb}_{0.97} \mathrm{La} 0.02\left(\mathrm{Zr}_{0.75} \mathrm{Sn}_{0.18} \mathrm{Ti}_{0.07}\right) \mathrm{O}_{3}$ AFE thick film, which is comparable with the best results in FE materials [19]. These results indicate that ECE originated from FE-AFE phase transition is a promising route for the practical cooling applications.

Energy-storage performance and ECE of the dielectric materials are strongly dependent on their polarization character, which could be tuned by the phase structure, grain size, stress, interface layer, growth orientation, and so on. Orientation is a powerful tool, through which, for instance, enhanced the energy-storage performance, tuned the ferroelectric-antiferroelectric phase transition, and improved the ECE [20-22]. Based on these results, in this work, we report that a typical lead-based AFE thick films of $\left(\mathrm{Pb}_{0.97} \mathrm{La}_{0.02}\right)\left(\mathrm{Zr}_{0.73} \mathrm{Sn}_{0.22} \mathrm{Ti}_{0.05}\right) \mathrm{O}_{3}$ exhibits an orientation-dependent energy storage performance and electrocaloric effect.

\section{Experimental procedure}

The composition of $(\mathrm{Pb0} 0.97 \mathrm{La0.02)}(\mathrm{Zr0} 0.73 \mathrm{Sn} 0.22 \mathrm{Ti0.05)O3}$ (PLZST) was selected to study, which is located in the morphotropic phase boundary (MPB). The PLZST precursor solution was deposited on Pt (111), LaNiO3(110)/Pt(111) and LaNiO3(10 0)/Pt(111) substrates via a sol-gel method, respectively. The detailed process could be found in reference [23]. The only difference is that the PLZST films deposited on Pt(111) was annealed at $700{ }^{\circ} \mathrm{C}$ for $25 \mathrm{~min}$ and the film deposited on $\mathrm{LaNiO} 3(110) / \mathrm{Pt}(111)$ and $\mathrm{LaNiO} 3(10$ 0)/Pt(111) were annealed at $700{ }^{\circ} \mathrm{C}$ for $30 \mathrm{~min}$ in order to reduce discrepancy of grain size. The final thickness of all the PLZST AFE thick film was about $1.5 \mathrm{~mm}$.

Here, LaNiO3 thin films were prepared on the $\mathrm{Pt}(111)$ by sol-gel method similar to Ref. [24]. Lanthanum nitrate $[\mathrm{La}(\mathrm{NO} 3) 3]$ and nickel acetate $[\mathrm{Ni}(\mathrm{CH} 3 \mathrm{COO}) 2.4 \mathrm{H} 2 \mathrm{O}]$ were used as the start 
materials, and acetic acid and water were used as the solvents. In order to avoid cracking during heating, formamide was also added to the system. The concentration of the precursor solution was adjusted to 0.2 M. The LaNiO3 precursor solution was spin coated on the $\mathrm{Pt}(111)$ substrates at $3000 \mathrm{rpm}$ for $20 \mathrm{~s}$. For LaNiO3(110) film, each wet film was heat treated at $450{ }^{\circ} \mathrm{C}$ for $10 \mathrm{~min}$. This procedure was repeated several times. The process was repeated several times. Finally, the LaNiO3 films were annealed at 700 ${ }^{\circ} \mathrm{C}$ for 60 min to obtain a well-crystallized structure. The whole thermal treatment was completed in a tube furnace [24]. For LaNiO3(100) film, each wet film was fired at $160{ }^{\circ} \mathrm{C}$ for $300 \mathrm{~s}$, then pyrolyzed at $400{ }^{\circ} \mathrm{C}$ for $360 \mathrm{~s}$ and finally annealed at higher temperatures for $240 \mathrm{~s}$. The process was repeated several times. Finally, the $\mathrm{LaNiO} 3$ films were annealed at $700{ }^{\circ} \mathrm{C}$ for $45 \mathrm{~min}$ to obtain desired thickness LaNiO3(100) films. The whole thermal treatment was completed in an RTP furnace [25]. The thickness of both $\mathrm{LaNiO} 3(110)$ film and $\mathrm{LaNiO} 3(100)$ film are about $400 \mathrm{~nm}$. In order to obtain the same grain size of both $\mathrm{LaNiO} 3$ (110) and $\mathrm{LaNiO} 3$ (100) films, the annealing time of LaNiO3 (100) film was shortened by 15 min than that in our previous work [23]. For convenience, the PLZST AFE thick films deposited on Pt(111), LaNiO3(110)/Pt(111) and LaNiO3(10 0)/Pt(111) substrates are abbreviated as PLZST(111), PLZST(110), and PLZST(100) films, respectively.

The microstructure of the PLZST AFE thick films was analyzed by X-ray diffraction (XRD Bruker D8 Advanced Diffractometer, German) and field-emission scanning electron microscopy (FE-SEM ZEISS Supra 55, German), respectively. For the electrical measurements, gold pads of $0.20 \mathrm{~mm}$ in diameter were coated on the films surface as top electrodes by using a DC sputtering method. A computer-controlled Agilent E4980A LCR analyzer was employed for measuring the frequency, and temperature-dependent dielectric properties with the AC drive amplitude of $50 \mathrm{mV}$. The polarization-electric field hysteresis loops $(\mathrm{P}-\mathrm{E})$ at $1 \mathrm{kHz}$ and the leakage current characteristic of the films were measured by a Ferroelectric tester (Radiant Technologies, Inc., Albuquerque, NM).

\section{Results and discussion}

Fig. 1 shows the XRD patterns of PLZST AFE thick films grown on Pt(111), LaNiO3(110)/Pt(111) and LaNiO3(10 0)/Pt(111) substrates after annealing at $700{ }^{\circ} \mathrm{C}$. For convenience, the lattice indexes of the 
diffraction peaks are labeled as pseudocubic structure. All the thick films have crystallized into a pure perovskite phase without any other phases. Apparently, the PLZST film on the Pt(111) substrate shows well (111)-preferred orientation, while PLZST films on the LaNiO3(110)/Pt(111) and LaNiO3(10 0)/Pt(111) substrates display (110) and (100)-preferred orientations, respectively. In order to estimate the orientation degree of the PLZST films on three substrates, orientation factor $\mathrm{a}(111), \mathrm{a}(110),{ }^{\text {and }} \mathrm{a}(100)$ are given according to the following well known formulas [26]:

$$
\begin{gathered}
\alpha_{(111)}=\frac{I_{(111)}}{I_{(111)}+I_{(110)}+I_{(100)}}, \\
\alpha_{(110)}=\frac{I_{(110)}}{I_{(111)}+I_{(110)}+I_{(100)}}, \\
\alpha_{(100)}=\frac{I_{(100)}}{I_{(111)}+I_{(110)}+I_{(100)}} . \\
.(111) \ldots \text {.110) } \ldots \text {-1uv) }
\end{gathered}
$$

The calculated values of $\alpha(111), \alpha(110)$, and $\alpha(100)$ are $0.95,0.86$, and 0.90 for PLZST(111), PLZST(110), and PLZST(100) films, respectively. For the PLZST(111) thick film, a transient intermetallic phase Pt3Pb with preferred-(111) orientation formed at the interface between the PLZST film and Pt(111) substrates during the heat treatment, which function as a seed layer and decrease the activation energy for crystallization of the film [27]. For both PLZST(110) and PLZST (100) films, the introduced LaNiO3 layer prevented the formation of intermetallic phase Pt3Pb between the PLZST film and Pt(111) substrates [28]. Meanwhile, the (110) and (100)preferred LaNiO3 buffer layer facilitate the growth of PLZST films along the (110) and (100) orientations, respectively, which is due to the same pseudocubic perovskite structure and the small lattice mismatch between AFE thick films and $\mathrm{LaNiO} 3$ bottom electrodes. The similar works were also reported in other lead-based FE and AFE films deposited on LaNiO3 bottom electrodes [29,30].

The FE-SEM images of surface and cross-sectional morphologies of all the films are given in Fig. 2(a-f). Evidently, these thick films show a dense, uniform and compact structure, and no micro-cracks or other microstructural defects are found in the films, which are attributed to the two-step heat-treatment procedure for the 
thick-film preparation. The average grain size is about 473, 467 and $468 \mathrm{~nm}$ for AFE thick films deposited on $\mathrm{Pt}(111)$, LaNiO3(110)/ $\mathrm{Pt}(111)$ and $\mathrm{LaNiO} 3(100) / \mathrm{Pt}(111)$ substrates, respectively, which are calculated by using the Nano Measurer software. The cross-sectional morphologies of these thick films present a columnarlike structure, indicating that the nucleation and growth of the crystals may initiate from the bottom electrodes [31]. The thickness of these films is the same and about $1.5 \mu \mathrm{m}$.

Fig. 3 displays the frequency dependence of dielectric constant and dielectric loss of the PLZST(111), PLZST(110) and PLZST(100) AFE thick films measured from $1 \mathrm{kHz}$ to $1 \mathrm{MHz}$ at room temperature. In the measurement frequency range, dielectric constant of three samples are decreased slightly because polarization process of some framework, such as space charges, needs longer time and thus contributes less to the overall polarization under higher frequencies [32]. All the samples share the low loss tangent of below 0.013, which is attributed to their uniform microstructure. It can be seen that the orientation of the films have a clear effect on their dielectric constant. The dielectric constant at $100 \mathrm{kHz}$ is 709 , 620, and 512 for the PLZST(111), PLZST (110) and PLZST(100) AFE thick films, respectively. This result is consistent to the dielectric constant at room temperature in $\mathrm{PbZrO} 3$ thin films [33].

Fig. 4 presents the temperature-dependent dielectric constant and dielectric loss of the PLZST(111), PLZST(110) and PLZST(100) AFE thick films measured at $100 \mathrm{kHz}$ and on heating rate of $3{ }^{\circ} \mathrm{C} / \mathrm{min}$. Obviously, the dielectric constant initially increase and then decrease with the further increase of temperature. The dielectric peaks corresponding to the transition from AFE to PE phase is observed at 184 ${ }^{\circ} \mathrm{C}$, 195, and $190{ }^{\circ} \mathrm{C}$ for the PLZST(111), PLZST(110) and PLZST(100) films, respectively, which correspond to the so-called Curie temperature $\left(\mathrm{T}_{\mathrm{c}}\right)$. The difference of the Curie temperature of the films should be caused by the difference in the substrate constraint and annealing procedure [34].

Room temperature P-E loops of all the samples are shown in Fig. $5(\mathrm{a}-\mathrm{c})$, which were measured at 1 kHz. Clearly, all the thick films exhibit double loops, demonstrating their AFE character. A small remnant polarization blow $5.0 \mathrm{pLC} / \mathrm{cm}^{2}$ is detected in these samples, which should be caused by the interface layer, space charge, residual ferroelectric state, unstable AFE regions, and so on. Under the measurement condition, the corresponding maximum polarization for the PLZST(111), PLZST(110) and PLZST(100) films is 77.3, 66.2 and $59.1 \mathrm{pLC} / \mathrm{cm}^{2}$, respectively. The large Pmax implies that a high recyclable energy- 
storage density and a large ECE could possibly be obtained in these films. The schematic diagram of the primitive cells in AFE and FE phases with different orientations is shown in Fig. $5(\mathrm{~d}-\mathrm{f})$. The polar direction of the antiparallel dipoles is along the [110] direction of the tetragonal primitive cell. When AFE phase changed into the FE phase under the applied electric field, the tetragonal primitive cell becomes rhombohedral with the polar directions along the [111] direction. Therefore, the PLZST(111) film possesses highest Pmax, followed by the PLZST(110), and then the PLZST(100). This result is consistent to that in PbZrO3 antiferroelectric films, which can be explained by that the oriented film with the higher Pmax is nearer to polar vector of FE phase hence leading to lower transition field $[21,29,35]$.

The energy-storage performance of the PLZST thick films with different crystallographic orientations are presented in Fig. 6(a), which were measured from $200 \mathrm{kV} / \mathrm{cm}$ to $900 \mathrm{kV} / \mathrm{cm}$ at $1 \mathrm{kHz}$ and at room temperature. As desired, for all the films, W values increase nearly linearly with increasing electric field. In the measurement range, the discoverable energy-storage density of the films is improved by controlling the orientations, which attribute to the high $\mathrm{P}_{\max }$. For example, the $\mathrm{W}$ value is $13.5,12.9$, and $11.6 \mathrm{~J} / \mathrm{cm}^{3}$ for the PLZST(111), PLZST(110) and PLZST(100) AFE thick films at $900 \mathrm{kV} / \mathrm{cm}$, respectively. In practical applications, larger energy-storage efficiency iri is also desired. With the increase of the measured electric field, the iri values are slightly declined and similar for these thick films. For example, the iri value of PLZST(111) thick film is changed from $73 \%$ to $63 \%$ as the electric field increases from 200 to $900 \mathrm{kV} / \mathrm{cm}$. To investigate the temperature-dependent stability of the capacitors, the energy-storage density and the energy-storage efficiency measured at $1 \mathrm{kHz}$ in the temperature ranging from 20 to $150{ }^{\circ} \mathrm{C}$ are presented in Fig. 6(b). In order to avoid electric breakdown at higher temperature, the measurements of P-E loops for all the samples are carried out at a lower electric field of $\mathrm{E}=900 \mathrm{kV} / \mathrm{cm}$. Evidently, all the curves only slightly vary in the measurement range, indicating good temperature stability of the energy-storage performance.

Assuming the Maxwell relation $\left(\frac{\partial P}{\partial T}\right)_{E}=\left(\frac{\partial S}{\partial E}\right)_{T}$, the reversible changes of $\Delta \mathrm{T}$ and $\Delta \mathrm{S}$ in a material at applied electric-field E can be expressed as [36]: 


$$
\begin{aligned}
& \Delta S=-\frac{1}{\rho} \int_{E_{1}}^{E_{2}}\left(\frac{\partial P}{\partial T}\right)_{E} \mathrm{~d} E, \\
& \Delta T=-\frac{1}{C \rho} \int_{E_{1}}^{E_{2}} T\left(\frac{\partial P}{\partial T}\right)_{E} d E,
\end{aligned}
$$

where $\mathrm{p}$ is density, $\mathrm{C}$ is themolar heat capacity, $\mathrm{T}$ is operating temperature, $\mathrm{P}$ is maximum polarisation at applied electric field E, E1 and E2 are the initial and final applied electric field, and $\left(\frac{\partial P}{\partial T}\right)$ is the pyroelectric effect at selected electric field. According to Eq. (8), the reversible adiabatic temperature changes AT of the PLZST(111), PLZST(110) and PLZST(100) AFE thick films at $900 \mathrm{kV} / \mathrm{cm}$ are plotted in Fig. 7(a). Here E1 $=0$ and $\mathrm{E} 2=\mathrm{E}$; thus $\mathrm{AE}$ is equal to $\mathrm{E}$. The specific heat capacity $\mathrm{C}=330 \mathrm{~J} \mathrm{~K}^{-1} \mathrm{~kg}^{-1}$ and the theoretical density $\mathrm{p}=8.3 \mathrm{~g} \mathrm{~cm}^{-3}$ are selected for these thick films as reported before [37]. Obviously, three samples display the same changing tendency in the operating temperature-dependent AT curves. As expected, the large AT of these films are received in a wide temperature range of room temperature, which is caused electric fieldinduced phase transition of FE-AFE. For example, the values of AT at $21{ }^{\circ} \mathrm{C}$ is $28.1,23.1$, and $21.2{ }^{\circ} \mathrm{C}$ for the PLZST(111), PLZST(110) and PLZST (100) films, respectively. With the operating temperature increasing, the AT firstly decrease, indicating a reduction of entropy change between AFE and FE phase transition. With the further increase of temperature, a peak of AT $=13.9,11.8$, and $10.7{ }^{\circ} \mathrm{C}$ is detected at 150,165 , and $169^{\circ} \mathrm{C}$ of the PLZST(111), PLZST and PLZST(100) films at $\mathrm{E}=900 \mathrm{kV} / \mathrm{cm}$, respectively, which is believed to be induced by the AFE-PE phase transition. The PLZST film displays the largest AT values, the PLZST(100) film has the lowest values, which coincides with the result in $\mathrm{PbTiO} / \mathrm{SrTiO} 3$ multilayers with different orientations [20]. The corresponding adiabatic AS of the PLZST(111), PLZST(110) and PLZST(100) films are given in Fig. 7(b), which were measured at $900 \mathrm{kV} / \mathrm{cm}$. Obviously, the changing tendency of AS is in agreement with AT, as a function of temperature. The maximum values of AS are 31.6, 25.9, and $23.8 \mathrm{~J} \mathrm{~K}^{-1} \mathrm{~kg}^{-1}$ of the PLZST(111), PLZST(110) and PLZST (100) films at room temperature at $900 \mathrm{kV} / \mathrm{cm}$, respectively. For the cooling applications, apart from large AT and AS, higher coefficient of performance COP $(C O P=\mathrm{jDS} \times T \mathrm{j}=\mathrm{j} W \mathrm{j})$ and electrocaloric coefficient (the ratio of AT/AE) are also desired [38,17]. The maximum COP and AT/AE of 5.7, 5.0, 4.9 and 0.031, 
0.026, 0.024 is achieved at room temperature and at $900 \mathrm{kV} / \mathrm{cm}$ in the PLZST(111), PLZST(110) and PLZST(100) films, respectively, as shown in Table 1.

Fig. 8(a) illustrates the current-time characteristics of the PLZST (111), PLZST(110) and PLZST(100) AFE thick films, as a function of external electric field, which were measured at room temperature and $600 \mathrm{kV} / \mathrm{cm}$. The decay in dielectric relaxation current density obeys the Curie-von Schweidler law as follows [39]:

$$
J=J_{s}+J 0 \times t^{-n}
$$

where $\mathrm{J}_{\mathrm{s}}$ is the steady-state current density, $\mathrm{J} 0$ a fitting constant, $\mathrm{t}$ the relaxation time in second, and $\mathrm{n}$ the slope of the log-log plot. It is believed that three possible mechanisms are associated with the Curie-von Schweidler law: space charge trapping, relaxation time distribution and electrical charge hopping [40]. By fitting the leakage current density data into Eq. (9), the steady-state leakage current density $\mathbf{J}_{\mathrm{s}}$ is $2.14 \times 10^{-6}$, $4.37 \times 10^{-6}$, and $5.41 \times 10^{-6} \mathrm{~A} / \mathrm{cm}^{2}$ for the PLZST(111), PLZST(110) and PLZST(100) films, respectively, as shown in Fig. 8(b). The smaller leakage current density is in agreement with our previous reports on the leadbased AFE films [4], which yield negligible Joule heating $\left(-10^{-3} \mathrm{~K}\right)$ and do not affect $\mathrm{P}-\mathrm{E}$ results because currents of hundreds of $\mathrm{mA}$ are required to switch the measured polarizations at $1 \mathrm{kHz}$. As a typical example, the current density-time characteristics of the PLZST(111) thick films are presented in Fig. 8(c), which was measured at $25,75,150$, and $200{ }^{\circ} \mathrm{C}$ and at $600 \mathrm{kV} / \mathrm{cm}$. The obtained steady-state leakage current density is $1.36 \times 10^{-6}, 3.47 \times 10^{-6}, 1.09 \times 10^{-5}$ and $2.29 \times 10^{-5} \mathrm{~A} / \mathrm{cm}^{2}$ at $25,75,150$, and $200{ }^{\circ} \mathrm{C}$, respectively. It could be obtained from these results that the leakage current density of the samples is increased as temperature increasing. The data could be fitted into the Arrhenius relationship [41]:

$$
J_{s}=\operatorname{Cexp}\left(-\frac{E_{a}}{k T}\right)
$$

where $\mathrm{C}$ is a fitting constant, $\mathrm{E}_{\mathrm{a}}$ the activation energy, and $\mathrm{k}$ the Boltzmann constant. According to the fitting curve showed in Fig. 8(d), the obtained activation energy is about $0.24 \mathrm{eV}$, which is close to the reported result in $\mathrm{Pb} 0.92 \mathrm{La} 0.08(\mathrm{Zr0} .52 \mathrm{Ti0} .48) \mathrm{O} 3$ film [42].

\section{Conclusion}


The energy-storage performance and cooling behavior were studied in (Pb0.97La0.02)(Zr0.73Sn0.22Ti0.05)O3 AFE thick films via orientation control. Because of its larger polarization in the thick films with (111) orientation, the best results of both energy-storage performance and ECE are simultaneously realized. A large recoverable energy-storage density of $13.5 \mathrm{~J} / \mathrm{cm}^{3}$ and corresponding efficiency of $73 \%$ were obtained at 900 $\mathrm{kV} / \mathrm{cm}$ in the PLZST AFE thick film with (111) orientation. A giant ECE value of DT $=28.1{ }^{\circ} \mathrm{C}$ was also achieved at $21{ }^{\circ} \mathrm{C}$ in the PLZST(111) AFE thick films during the FE-AFE switching, and the corresponding DS was $31.6 \mathrm{~J} \mathrm{~K}-1 \mathrm{~kg}^{-1}$. Moreover, a smaller leakage current density was detected in these AFE thick films, which is favored to their applications. In summary, these results indicate that AFEs are promising candidates for both energy-storage capacitors and room-temperature cooling devices, and the performance could be enhanced by the proper orientation control.

\section{Acknowledgements}

The authors would like to acknowledge the financial support from the Ministry of Sciences and Technology of China through 973-project (2014CB660811), the National Natural Science Foundation of China (51462027), the Program for Innovative Research Team in Universities of Inner Mongolia Autonomous Region (NMGIRT-A1605), the Innovation Lead Project of Boutao (CX5015-8) and the Innovation Program of Inner Mongolia University of Science and Technology (2014QNGG01).

\section{References}

[1] C.W. Ahn, G. Amarsanaa, S.S. Won, S.A. Chae, D.S. Lee, I.W. Kim, Antiferroelectric thin-film capacitors with high energy-storage densities, low energy losses, and fast discharge times, ACS Appl. Mater. Interfaces 48 (2015) 26381-26386.

[2] Q. Li, K. Han, M.R. Gadinski, G.Z. Zhang, Q. Wang, Energy storage: high energy and power density capacitors from solution-processed ternary ferroelectric polymer nanocomposites, Adv. Mater. 26 (2014) 6356-6356.

[3] Z.Q. Hu, B.H. Ma, R.E. Koritala, U. Balachandran, Temperature-dependent energy storage properties of antiferroelectric Pb0.96La0.04Zr0.98Ti0.02O3 thin films, Appl. Phys. Lett. 104 (2014) 263902.

[4] Y. Zhao, X.H. Hao, Q. Zhang, Energy-storage properties and electrocaloric effect of $\mathrm{Pb}(1-$ 3x/2)LaxZr0.85Ti0.15O3 antiferroelectric thick films, ACS Appl. Mater. Interfaces 6 (2014) 1163311639. 
[5] B. Peng, Z.K. Xie, Z.X. Yue, L.T. Li, Improvement of the recoverable energy storage density and efficiency by utilizing the linear dielectric response in ferroelectric capacitors, Appl. Phys. Lett. 105 (2014) 052904.

[6] Y.Y. Liu, Y. Wang, X.H. Hao, J.B. Xu, Preparation and energy-storage performance of PLZT antiferroelectric thick films via sol-gel method, Cerem. Int. 39 (2013) S513-S516.

[7] X.H. Hao, Y. Wang, L. Zhang, L.W. Zhang, S.L. An, Composition-dependent dielectric and energystorage properties of (Pb,La)(Zr,Sn,Ti)O3 antiferroelectric thick films, Appl. Phys. Lett. 102 (2013) 163903.

${ }_{[8]}$ B.L. Peng, Q. Zhang, X. Li, T.Y. Sun, H.F. Fan, S.M. Ke, M. Ye, Y. Wang, W. Lu, H.B. Niu, X.R. Zeng, H.T. Huang, Large energy storage density and high thermal stability in a highly textured (111)-oriented $\mathrm{Pb} 0.8 \mathrm{Ba} 0.2 \mathrm{ZrO} 3$ relaxor thin film with the coexistence of antiferroelectric and ferroelectric phases, ACS Appl. Mater. Interfaces 7 (2015) 13512-13517.

[9] X.H. Hao, J.W. Zhai, X. Yao, Improved energy storage performance and fatigue endurance of Sr-doped $\mathrm{PbZrO3}$ antiferroelectric thin films, J. Am. Ceram. Soc. 92 (2009) 1133-1135.

[10] M. Ye, P. Lin, H.T. Huang, B.L. Peng, Q. Sun, F.P. Wang, X. Peng, X.R. Zeng, S.M. Ke, PbZrO3Based antiferroelectric thin film capacitors with high energy storage density, Int. J. Adv. App. Phys. Res. 1 (2014) 35-39.

[11] M. Valant, Electrocaloric materials for future solid-state refrigeration technologies, Prog. Mater. Sci. 57 (2012) 980-1009.

[12] H.M. Gu, B. Craven, X.S. Qian, X.Y. Li, A.L. Cheng, Q.M. Zhang, Simulation of chip-size electrocaloric refrigerator with high cooling-power density, Appl. Phys. Lett. 102 (2013) 112901.

[13] X.S. Qian, H.J. Ye, Y.T. Zhang, H.M. Gu, X.Y. Li, C.A. Randall, Q.M. Zhang, Giant electrocaloric response over a broad temperature range in modified BaTiO3 ceramics, Adv. Funct. Mater. 24 (2014) 1300-1305.

[14] X. Moya, E. Stern-Taulats, S. Crossley, D. González-Alonso, S. Kar-Narayan, A. Planes, L. Mañosa, N.D. Mathur, Giant electrocaloric strength in single-crystal BaTiO3, Adv. Mater. 25 (2013) 1360-1365.

[15] B. Neese, S.G. Lu, B.J. Chu, Q.M. Zhang, Electrocaloric effect of the relaxor ferroelectric poly(vinylidene fluoride-trifluoroethylene-chlorofluoroethylene) terpolymer, Appl. Phys. Lett. 94 (2009) 042910.

[16] A.S. Mischenko, Q. Zhang, J.F. Scott, R.W. Whatmore, N.D. Mathur, Giant electricaloric effect in thin-film PbZr0.95Ti0.05O3, Science 311 (2006) 1270- 1271.

[17] A.S. Mischenko, Q. Zhang, R.W. Whatmore, N.D. Mathur, Giant electrocaloric effect in the thin film relaxor ferroelectric 0.9PbMg1/3Nb2/3O3-0.1PbTiO3 near room temperature, Appl. Phys. Lett. 89 (2006) 242912. 
P.D. Thacher, Electrocaloric effects in some ferroelectric and antiferroelectric $\mathrm{Pb}(\mathrm{Zr}, \mathrm{Ti}) \mathrm{O} 3$ compounds, J. Appl. Phys. 39 (1968) 1996-2002.

[19] Y. Zhao, X.H. Hao, Q. Zhang, A giant electrocaloric effect of a Pb0.97La0.02

(Zr0.75Sn0.18Ti0.07)O3 antiferroelectric thick film at room temperature, J. Mater. Chem. C 3 (2015) 1694-1699.

[20] J. Ge, D. Remiens, J. Costecalde, Y. Chen, X.L. Dong, G.S. Wang, Effect of residual stress on energy storage property in $\mathrm{PbZrO} 3$ antiferroelectric films with different orientations, Appl. Phys. Lett. 103 (2013) 162903.

[21] J. Ge, D. Remiens, X.L. Dong, Y. Chen, J. Costecalde, F. Gao, F. Cao, G.S. Wang, Enhancement of energy storage in epitaxial $\mathrm{PbZrO} 3$ antiferroelectric films using strain engineering, Appl. Phys. Lett. 105 (2014) 112908.

[22]J.H. Qiu, J.N. Ding, N.Y. Yuan, X.Q. Wang, J. Yang, Orientation dependence of the electrocaloric effect in PbTiO3/SrTiO3 multilayers, Solid State Commun. 152 (2012) 856-859.

[23] Y. Zhao, X.H. Hao, Q. Zhang, Improved electrocaloric effect in (100)-oriented $\mathrm{Pb} 0.97 \mathrm{La} 0.02(\mathrm{Zr} 0.57 \mathrm{Sn} 0.38 \mathrm{Ti0.05}) \mathrm{O} 3$ antiferroelectric thick film by interface engineering, ACS Appl. Mater. Interfaces 6 (2014) 11633-11639.

[24] X.H. Hao, J.W. Zhai, X. Yao, Improved dielectric properties of (110)-preferred (Pb,La)(Zr,Sn,Ti)O3 antiferroelectric thin films on metalorganic decomposition-derived LaNiO3 buffer layer, J. Cryst. Growth 311 (2008) 90-94.

[25]J.K. Li, X. Yao, Microstructure and electrical properties of $\mathrm{Pb}(\mathrm{Zr} 0.52 \mathrm{Ti0.48}) \mathrm{O} 3$ ferroelectric films on different bottom electrodes, Mater. Lett. 58 (2004) 3447-3450.

${ }_{[26]}$ X.H. Hao, J.W. Zhai, J.B. Xu, Xi Yao, Preparation of PLZT antiferroelectric thin films on ZrO2 buffered substrates, Ferroelectrics 357 (2007) 253-258.

[27] T. Tani, Z. Xu, D.A. Payne, Preferred orientations for sol-gel derived PLZT thin layers, Mater. Res. Sot. Symp. Proc. 310 (1993) 269-274.

[28] X.H. Hao, J.W. Zhai, X. Yao, Preparation of highly (111)-oriented (Pb,La)(Zr,Sn, Ti)O3 (PLZST) antiferroelectric thin films by modified sol-gel process using novel tin sourse, dibutyloxide of tin, J. Sol-Gel Sci. Technol. 42 (2007) 365-368.

[29] X.H. Hao, J.W. Zhai, J.C. Yang, H.P. Ren, X.W. Ren, Improved field-induced strains and fatigue endurance of PLZT antiferroelectric thick films by orientation control, Phys. Status Solidi RRL 3 (2009) $248-250$.

[30] X.H. Hao, J.W. Zhai, F. Shang, J. Zhou, S.L. An, Orientation-dependent phase switching process and strains of Pb0.97La0.02(Zr0.85Sn0.13Ti0.02)O3 antiferroelectric thin films, J. Appl. Phys. 107 (2010) 116101. 
[31] H.F. Ji, W. Ren, L.Y. Wang, P. Shi, X.F. Chen, X.Q. Wu, X. Yao, S.T. Lau, Q.F. Zhou, K. K. Shung, Structure and electrical properties of $\mathrm{Na} 0.5 \mathrm{Bi} 0.5 \mathrm{TiO} 3$ ferroelectric thick films derived from a polymer modified sol-gel method, IEEE Trans. Ultrason. Ferroelectr. Freq. Control 58 (2011) 2042-2049.

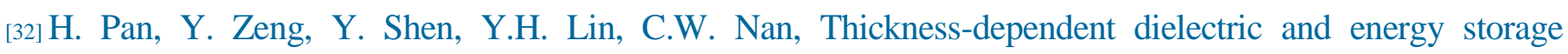
properties of $(\mathrm{Pb} 0.96 \mathrm{La0.04})(\mathrm{Zr} 0.98 \mathrm{Ti0.02}) \mathrm{O} 3$ antiferroelectric thin films, J. Appl. Phys. 119 (2016) 124106.

[33] T. Tani, J.F. Li, D. Viehland, D.A. Payne, Antiferroelectric-ferroelectric switching and induced strains for sol-gel derived lead zirconate thin layers, J. Appl. Phys. 75 (1994) 3017-3023.

[34] X.H. Hao, J.W. Zhai, J.B. Xu, X. Yao, Effect of orientation on the dielectric properties of Pb0.97La0.02Zr0.95Ti0.05O3 (PLZT) antiferroelectric thin films, Ferroelectrics 357 (2007) 218-222.

[35] B.M. Xu, Y.H. Ye, L.E. Cross, Dielectric properties and field-induced phase switching of lead zirconate titanate stannate antiferroeletric thick films on silicon substrates, J. Appl. Phys. 87 (2000) 2507-2515.

[36] B. Neese, B.J. Chu, S.G. Lu, Y. Wang, E. Furman, Q.M. Zhang, Large electrocaloric effect in ferroelectric polymers near room temperature, Science 321 (2008) 821-823.

[37] X.H. Hao, Z.X. Yue, J.B. Xu, S.L. An, C.W. Nan, Energy-storage performance and electrocaloric effect in (100)-oriented Pb0.97La0.02(Ti0.95Zr0.05)O3 antiferroelectric thick films, J. Appl. Phys. 110 (2011) 064109.

${ }^{[38]}$ E. Defay, S. Crossley, S. Kar-Narayan, X. Moya, N.D. Mathur, The electrocaloric efficiency of ceramic and polymer films, Adv. Mater. 25 (2013) 3337-3342.

[39] B.H. Ma, D.K. Kwon, M. Narayanan, U. Balachandran, Leakage current characteristics and dielectric breakdown of antiferroelectric Pb0.92La0.08Zr0.95Ti0.05O3 film capacitors grown on metal foils, J. Phys. D: Appl. Phys. 41 (2008) 205003.

[40] L. Zhang, X.H. Hao, Dielectric properties and energy-storage performances of (1-x)(Na0.5Bi0.5)TiO3xSrTiO3 thick films prepared by screen printing technique, J. Alloys Comp. 586 (2014) 674-678.

[41] B.H. Ma, S. Chao, M. Narayanan, S.S. Liu, S. Tong, R.E. Koritala, U. Balachandran, Dense PLZT films grown on nickel substrates by PVP-modified sol-gel method, J. Mater. Sci. 48 (2013) 1180-1185.

[42] S. Tong, B.H. Ma, M. Narayanan, S.S. Liu, R. Koritala, U. Balachandran, D.L. Shi, Lead lanthanum zirconate titanate ceramic thin films for energy storage, ACS Appl. Mater. Interfaces 5 (2013) 14741480. 


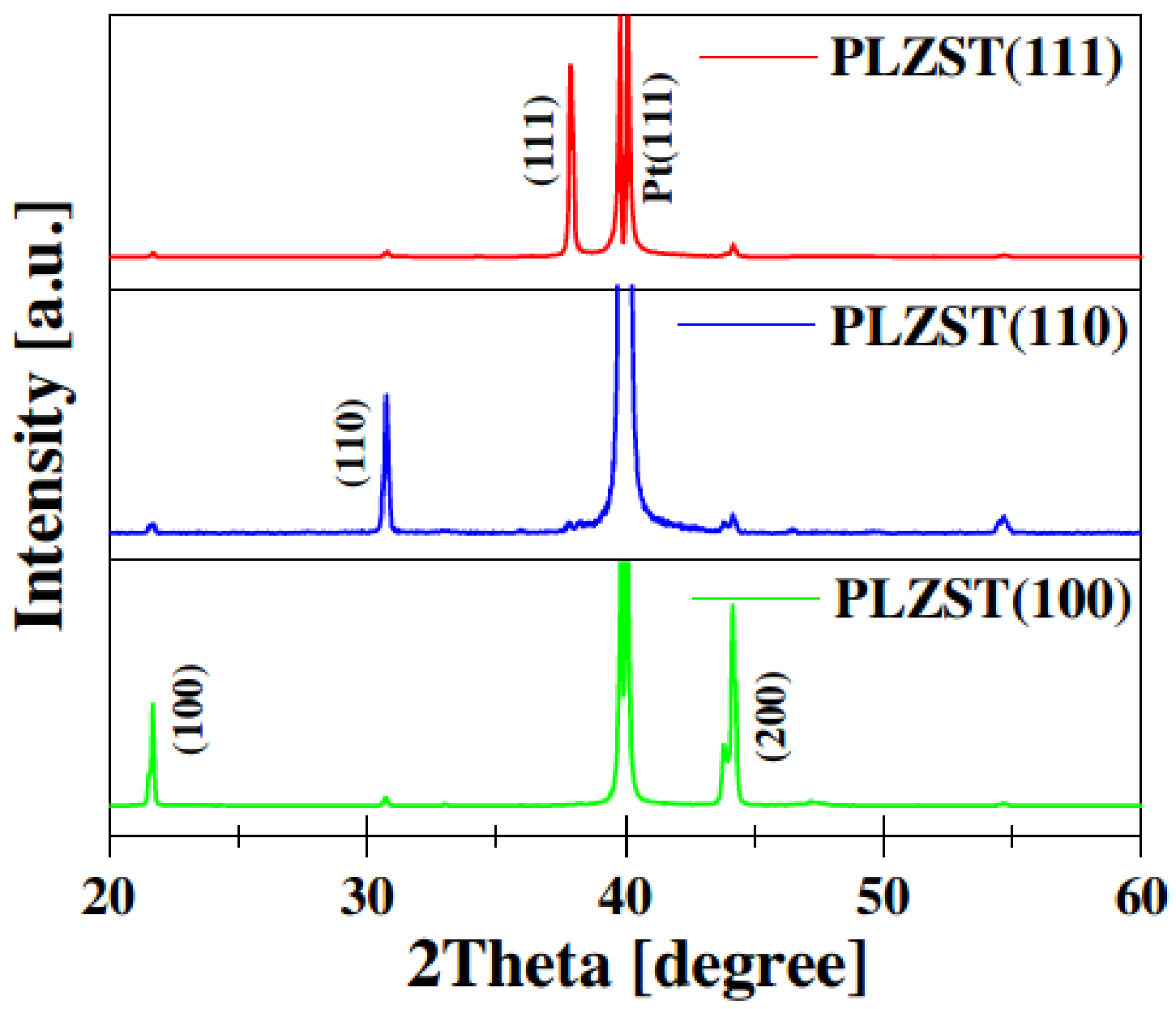

Fig. 1. XRD patterns of the PLZST(111), PLZST(110) and PLZST(100) AFE thick films. 


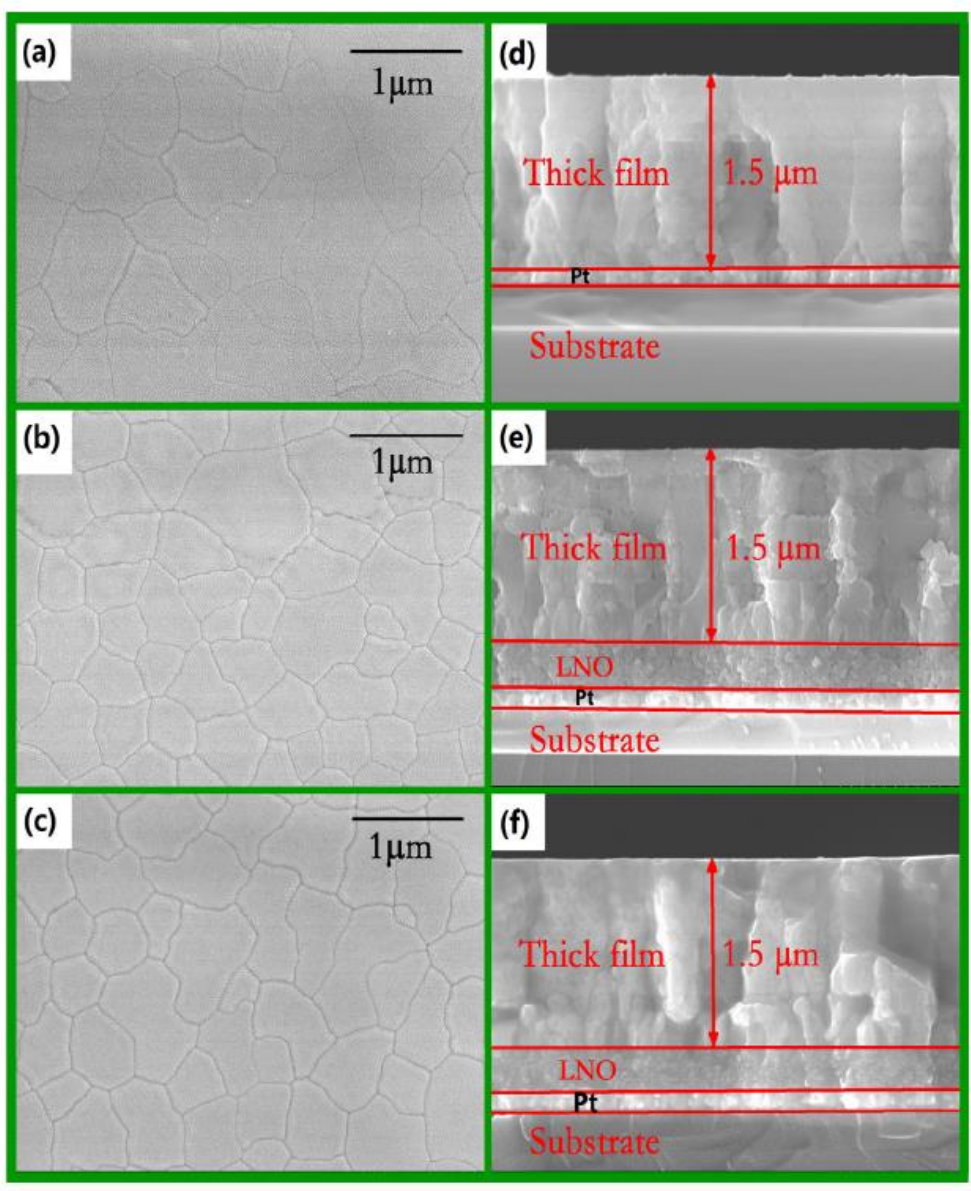

Fig. 2. Surface FE-SEM morphology images of the PLZST AFE thick films (a) PLZST(111), (b) PLZST(110), (c) PLZST(100); cross-sectional images of the films (d) PLZST(111), (e) PLZST(110), (f) PLZST(100). 


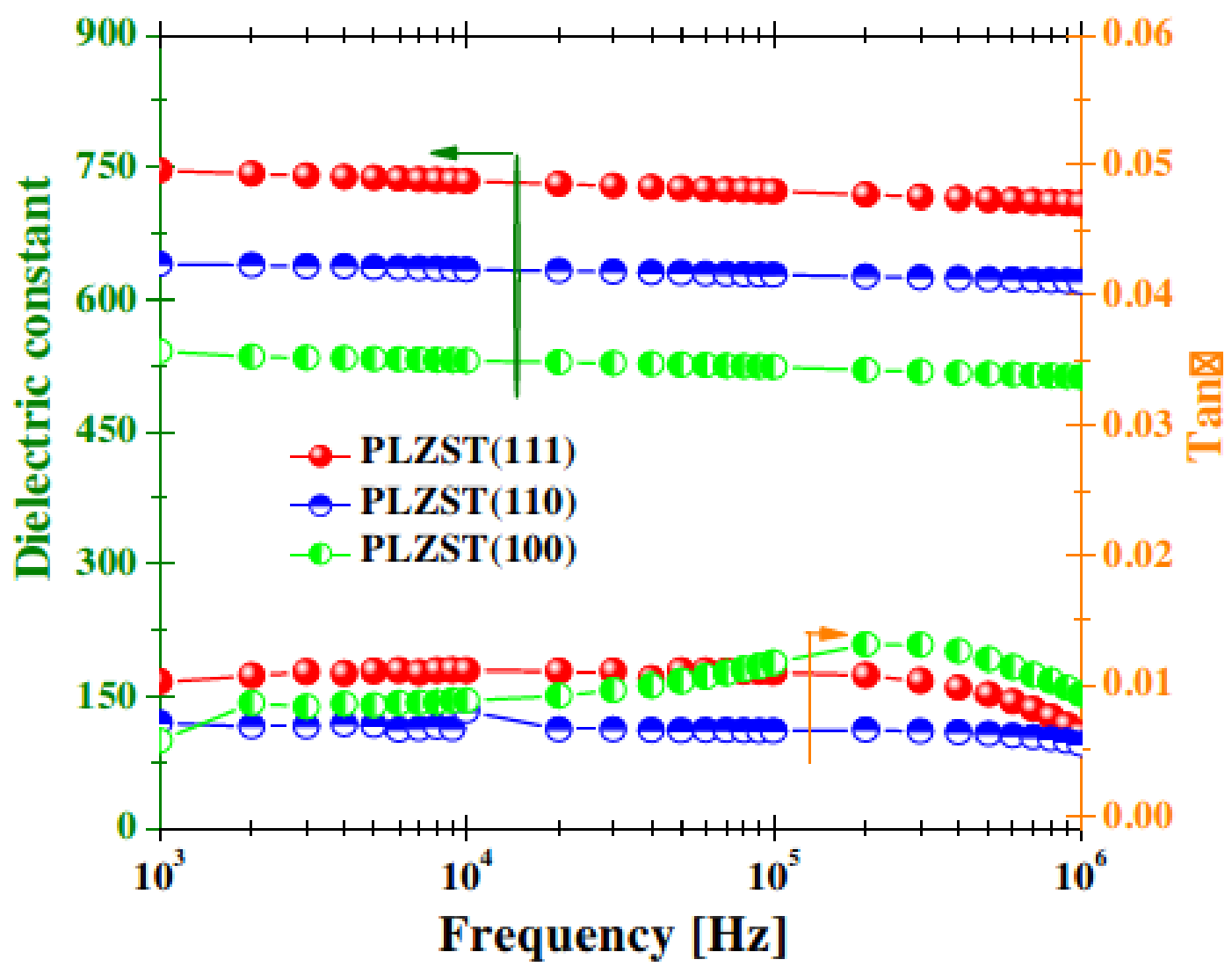

Fig. 3. Room temperature frequency-dependent dielectric constant and dielectric loss of the PLZST(111), PLZST(110) and PLZST(100) AFE thick films. 
(a) (b) (c)
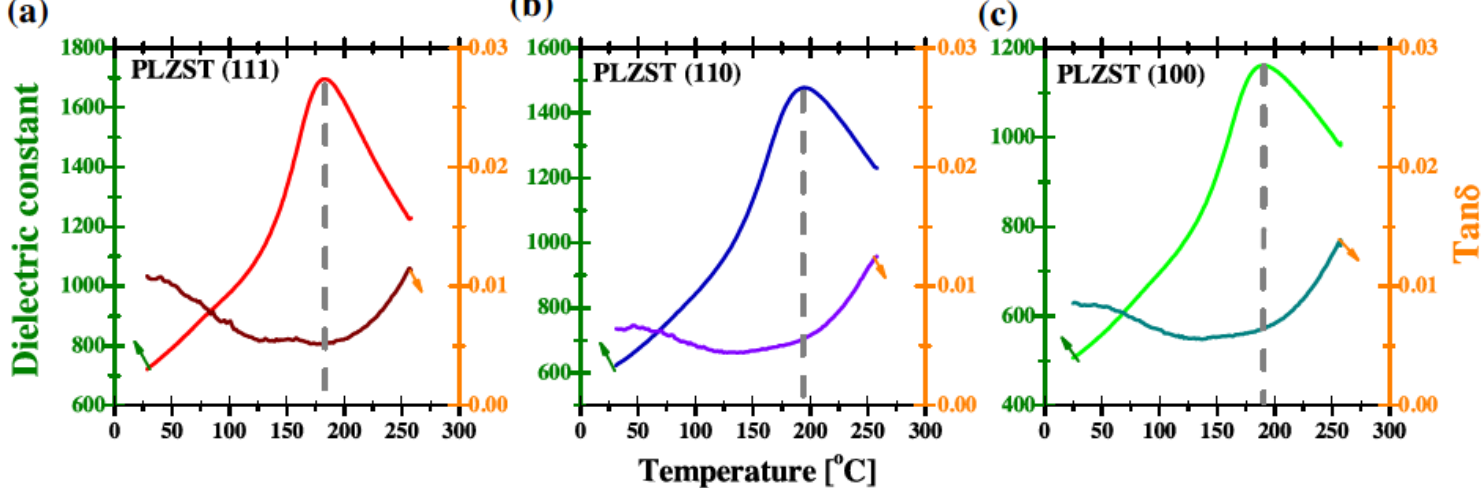

Fig. 4. Temperature dependence of dielectric constant and dielectric loss of the PLZST(111), PLZST(110) and PLZST(100) AFE thick films at 100 kHz. 


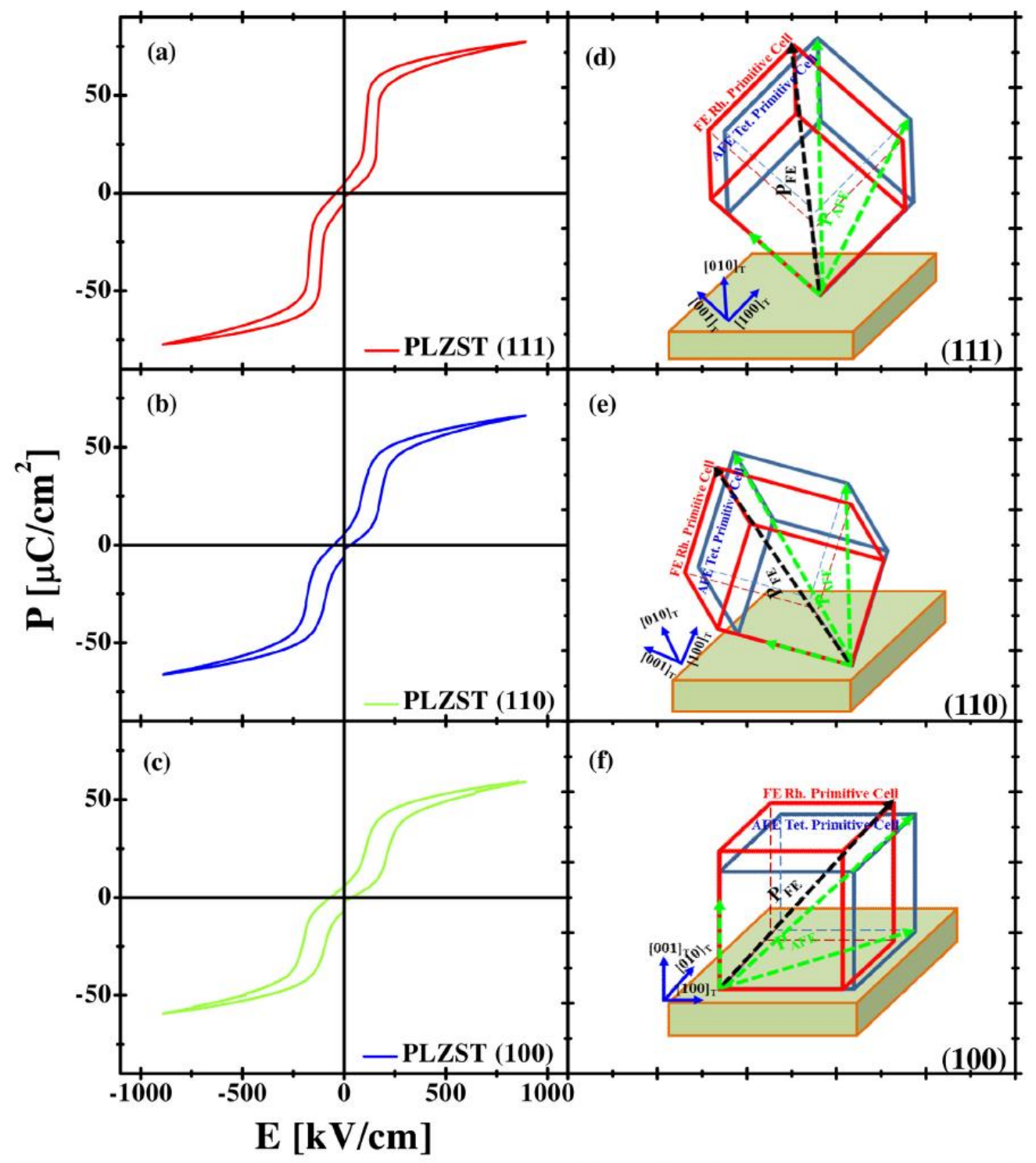

Fig. 5. (a-c) Room temperature P-E loops of the PLZST(111), PLZST(110) and PLZST(100) AFE thick films at $1 \mathrm{kHz}$. (d-f) the corresponding schematic diagram of the primitive cells. 

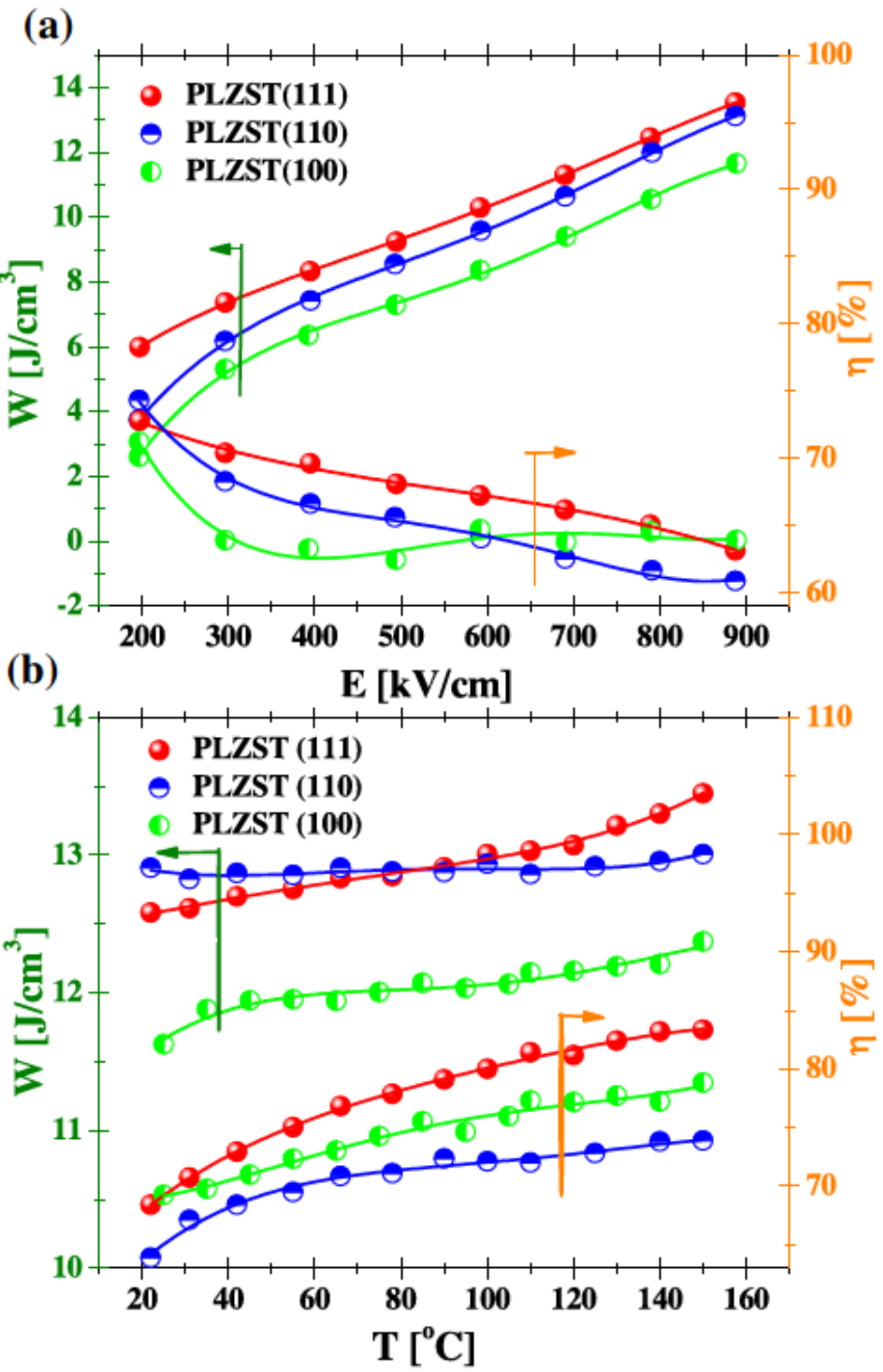

Fig. 6. (a) Electric-field dependence of recoverable energy-storage density and energy-storage efficiency of the PLZST(111), PLZST(110) and PLZST(100) AFE thick films. (b) The corresponding temperature dependence of energy-storage density and energy-storage efficiency measured at $900 \mathrm{kV} / \mathrm{cm}$. 

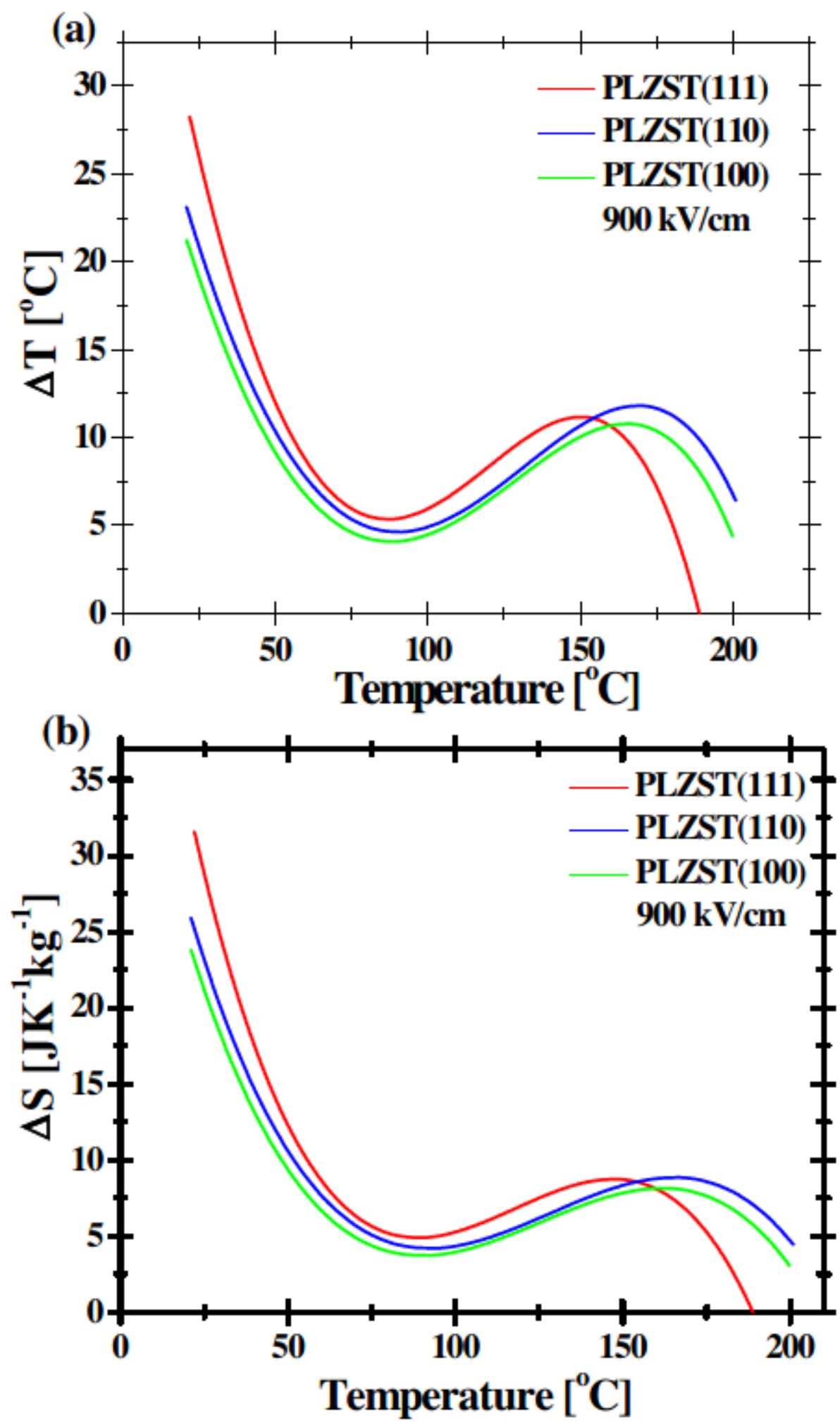

Fig. 7. (a) The adiabatic temperature changes $\Delta T$ as a function of temperature under $990 \mathrm{kV} / \mathrm{cm}$ of the PLZST(111), PLZST(110) and PLZST(100) AFE thick films, and (b) the corresponding adiabatic changes in entropy $\Delta \mathrm{S}$ of the films. 
(a)

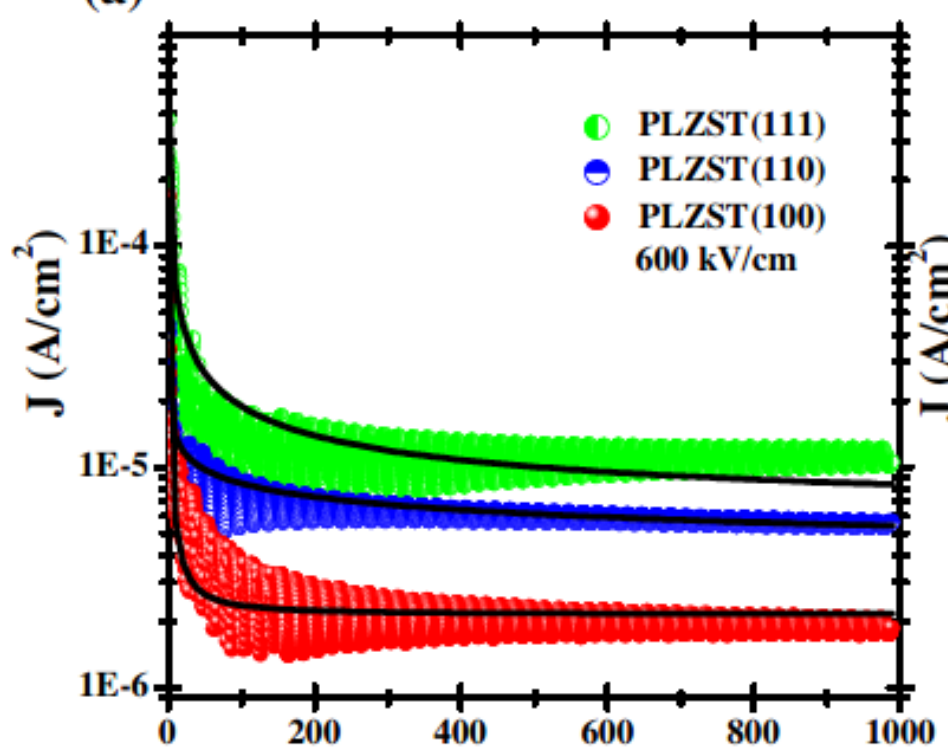

(b)

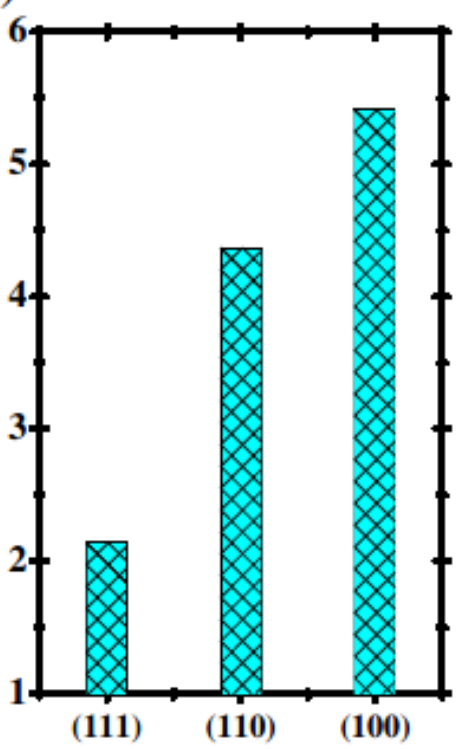

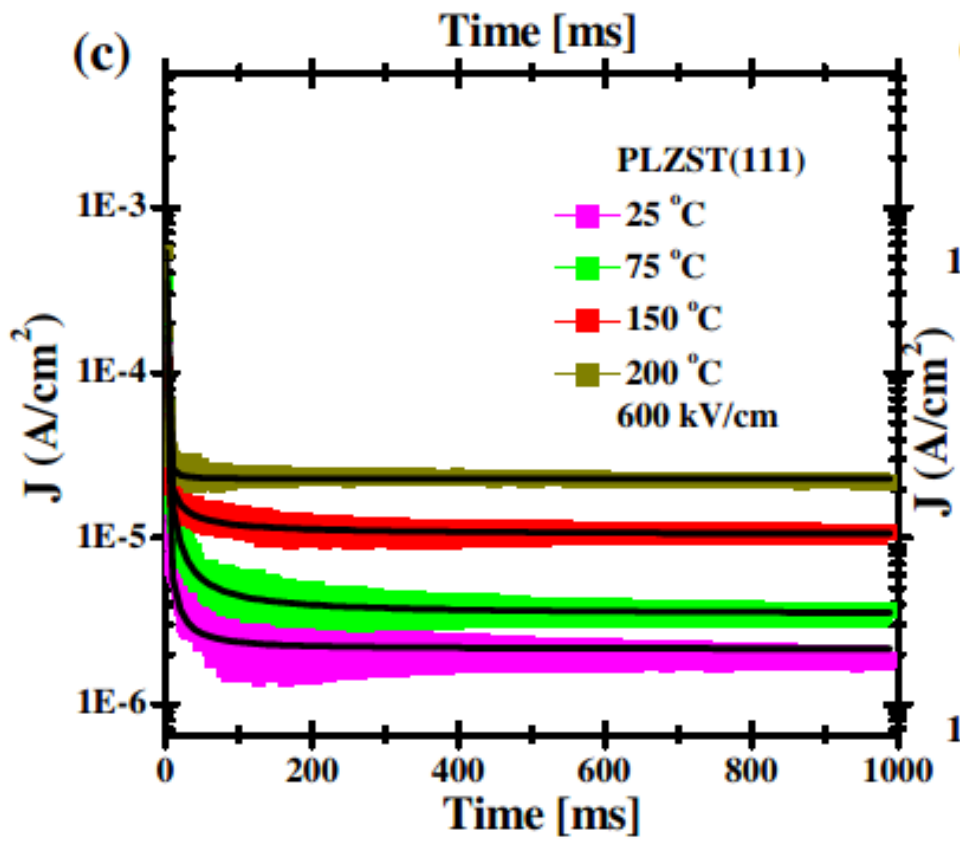

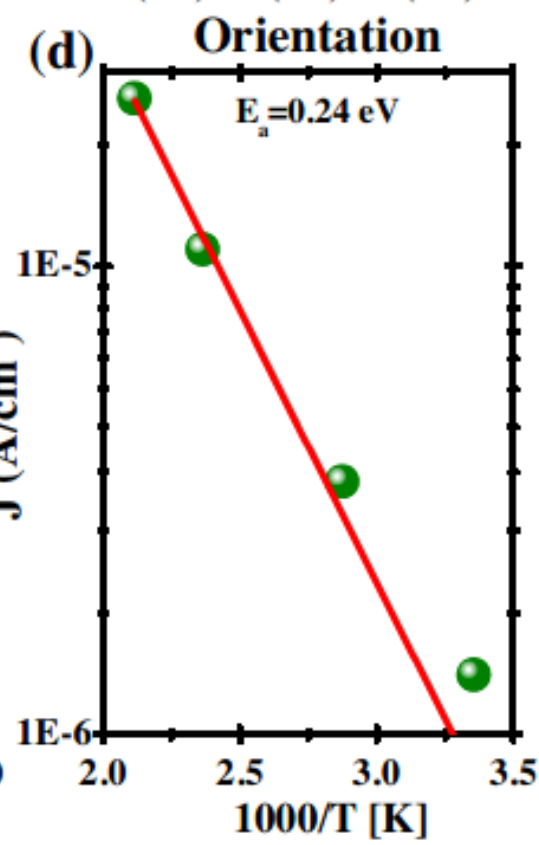

Fig. 8. (a) Dielectric relaxation current of the PLZST(111), PLZST(110) and PLZST(100) AFE thick films under $600 \mathrm{kV} / \mathrm{cm}$ and room temperature. (b) The corresponding steadystate leakage current of the films. (c) The temperature-dependent dielectric relaxation current and corresponding fitting curve of the PLZST(111) film at different temperature. (d) The corresponding steady-state current density and corresponding fitting curve as a function of reciprocal temperature. 
Table 1

The parameters on ECE of PLZST thick films with different orientation at room temperature.

\begin{tabular}{lllll}
\hline Orientation & $\mathrm{COP}$ & $\xi_{\max }(\mathrm{Kcm} / \mathrm{kV})$ & $\Delta \mathrm{T}\left({ }^{\circ} \mathrm{C}\right)$ & $\Delta \mathrm{S}\left(\mathrm{JK}^{-1} \mathrm{~kg}^{-1}\right)$ \\
\hline$(111)$ & 5.7 & 0.031 & 28.1 & 31.6 \\
$(110)$ & 5.0 & 0.026 & 23.1 & 25.9 \\
$(100)$ & 4.9 & 0.024 & 21.2 & 23.8 \\
\hline
\end{tabular}




\section{Orientation-dependent energy-storage performance and electrocaloric effect in PLZST antiferroelectric thick films}

Zhao, Y.

Elsevier

Zhao Y, Gao H, Hao X, Zhang Q, Orientation-dependent energy-storage performance and electrocaloric effect in PLZST antiferroelectric thick films, Materials Research Bulletin, Volume pÿ84, December 2016, Pages 177184.

http://dx.doi.org/10.1016/j.materresbull.2016.08.005

Downloaded from Cranfield Library Services E-Repository 